\title{
Studies on Convulsive Disorders in Young Children
}

\author{
I. Incidence of Febrile and Nonfebrile Convulsions by Age and Other Factors
}

Bea J. van DEn BerG ${ }^{[16]}$ and J.Yerushalmy

\begin{abstract}
The Child Health and Development Studies of the Division of Biostatistics, School of Public Health, University of California, Berkeley, Galifornia;

the Permanente Medical Group and the Kaiser Foundation Research Institute, Oakland, California, USA
\end{abstract}

\section{Extract}

In a cohort of 18,500 newborn infants, those who had experienced one or more convulsions were studied. Data were available for the unaffected as well as for the affected children. All families were members of a prepaid medical care program that provides comprehensive medical care.

By five years of age, two percent of the 18,500 children had had one or more febrile convulsions; one percent had had nonfebrile convulsions. The incidence of the first nonfebrile seizure was highest during the first month of life; febrile convulsions reached a high peak during the second year of life.

The risk of a febrile convulsion was equal for boys and girls but that of a nonfebrile seizure was higher for boys. The nonfebrile group had an excess of children with birth weights between 1500 and $2500 \mathrm{~g}$, especially those whose gestational age was 37 weeks or more. The distribution by birth weight and gestational age of the febrile group was similar to that of the entire cohort.

Multiple episodes occurred in one-third of the children of the febrile group and in three-quarters of the nonfebrile group. About $3 \%$ of the children with febrile seizures later developed nonfebrile convulsions; about $7 \%$ of the nonfebrile group had initially a febrile seizure. Nearly $31 \%$ in the nonfebrile group and $7 \%$ in the febrile group had severe congenital anomalies; in the entire cohort only $3 \%$ had such an anomaly before they reached age five. Among the children in the nonfebrile group were $10 \%$ with cerebral palsy and $16 \%$ with mental retardation including $5 \%$ with both disorders.

\section{Speculation}

As the cohort of 18,500 children grows older, a certain number of them will develop real epilepsy. This study provides three distinct groups: children who had febrile convulsions, those who had nonfebrile convulsions, and those who had no seizures in early childhood. The future follow-up of these groups will provide the necessary data for determining the relative rates of developing epilepsy associated with the convulsion experience in early childhood. 


\section{Introduction}

The unique features of these studies are first, that they relate to all the children who experienced one or more convulsions among a large cohort of newborn infants, and second, that data are available for the unaffected as well as for the affected children. In the course of an extensive investigation, in which a large number of pregnant women and their children were observed and followed longitudinally, all children who had experienced one or more convulsive seizures were identified and studied. Data on biologic, medical and environmental factors were obtained prospectively on all the families of the cohort. Data were also available on the course of pregnancy, labor, and delivery.

In this setting, it is possible to provide fairly reliable incidence rates and to investigate the relation of convulsive disorders to the many factors that are available for the entire cohort. In addition, information on treatment and prognosis becomes available as the follow-up of the children continues. It is hoped, therefore, that these studies will provide data from which the dynamics of the different manifestations of these disorders may be explored. The present paper is limited to the provision of estimates of the incidence of febrile and nonfebrile convulsions by age, sex, birth weight and gestational age, and to the investigation of the possible relation of convulsive disorders to severe congenital anomalies.

\section{Material and Method}

The cohort was composed of some 18,500 children born alive between 1960 and 1967 in Kaiser Foundation Hospital, Oakland, California. The parents were members of the Kaiser Health Plan, a prepaid medical care program in the San Francisco-East Bay area that provides comprehensive medical in- and out-patient services for the entire family. The women reported for prenatal care relatively early in their pregnancy. At the first visit an extensive and comprehensive interview was obtained. Participation of the pregnant women in the Child Health and Development Studies was nearly $100 \%$.

The population was broadly based and typical of an employed population. It is deficient only in very indigent and very affluent segments. Socio-economically and educationally, the study population was of a somewhat higher level than that of the general San Francisco-East Bay area. Ethnically, $65 \%$ of the population were white, $23 \%$ black, and the remainder were of other or mixed ethnic backgrounds.

Information on illnesses and medication during pregnancy was derived from the medical records of the obstetric and other specialty clinics of the Kaiser Hospitals in the East Bay Area. The children were observed regularly in pediatric clinics. The records of visits to the out-patient pediatric clinics and to all other specialty clinics, as well as the records for any periods of hospitalization, are available in one comprehensive file.

The pediatrician could not often observe the child during a seizure; however, the child was nearly always seen shortly after an episode. At that time great effort was exerted to obtain from the mother a detailed history and description of the attack. This enabled the pediatrician to ascertain whether the episode was indeed a convulsive disorder. Since comprehensive inand out-patient care was available for the entire family, it is unlikely that many definite convulsions were unreported.

Episodes of convulsive disorders may occur concurrently with other conditions, or the seizure may be the only observable phenomenon. Most frequently convulsions in the age groups studied occur concurrently with high fever and systemic infection. Such convulsions are referred to as febrile. Convulsive disorders that occur in the absence of fever are combined under the heading of nonfebrile.

Occurrences of convulsive disorders stimulated a number of diagnostic activities (table I). About onehalf of the children having nonfebrile seizures were hospitalized; more than one-half received out-patient neurologic consultation in addition to consultations during hospitalization; more than one-fourth had an x-ray examination of the skull; and nearly two-fifths had an EEG examination. Even among the febrile group, nearly one-quarter was hospitalized and about one-quarter had a spinal fluid examination.

Fifty percent of the initial nonfebrile seizures were generalized myoclonic or the 'grand mal' type; twelve

Table I. Diagnostic procedures for children with febrile and nonfebrile convulsive disorders

\begin{tabular}{lcc}
\hline Procedures & \multicolumn{2}{c}{ Percent of children } \\
\cline { 2 - 3 } & Febrile & Nonfebrile \\
\hline Hospitalizations & 24.4 & 52.2 \\
Neurologic consultation ${ }^{1}$ & 5.7 & 54.0 \\
X-ray skull & 7.7 & 28.3 \\
EEG & 6.9 & 38.0 \\
Echogram & 0.8 & 7.0 \\
Spinal fluid & 28.0 & 19.4 \\
Ventriculogram & - & 10.6 \\
Number of children & 246 & 113 \\
\hline
\end{tabular}

1 In out-patient clinic. 
percent were localized myoclonic; about the same percent were mainly hypertonic; and twenty percent were akinetic episodes with loss of posture or spells of staring. Two percent of the children had typical symptoms and signs of 'petit mal' and three percent had 'infantile spasms'. Febrile seizures, however, were more uniform. Nearly ninety percent of the first febrile seizures were generalized myoclonic and the remainder were mainly hypertonic or hypotonic spells.

\section{Incidence of Convulsive Disorders}

In calculating the incidence rates, it is necessary to take into consideration the fact that the length of follow-up was not the same for all the children. The youngest, born in 1967, have been followed for only one year while the oldest, born in 1960, have been followed for more than seven years. The average age of the children, when last observed, was 3.2 years. The incidence rates, therefore, were calculated on the basis of the number of children who were actually observed during each period.

The differences in incidence by ethnic group were very small; therefore, the data are combined for all groups.

\section{Age at first seizure}

Among the 18,500 children, 359 had experienced one or more convulsions. The average monthly incidence rate, by age at first attack, was dissimilar for febrile and nonfebrile disorders (table II and fig. 1).
Febrile convulsions were rare in the first few months of life, increased and reached a peak in the second year of life, dropped sharply in the third year and slowly thereafter. Nonfebrile disorders, however, may occur almost immediately after birth. The monthly incidence of the first seizure of the nonfebrile disorders reached a peak in the first month of life and dropped sharply thereafter.

Figure 2 shows cumulative incidence rates. By the age of five years, about two percent of the children had

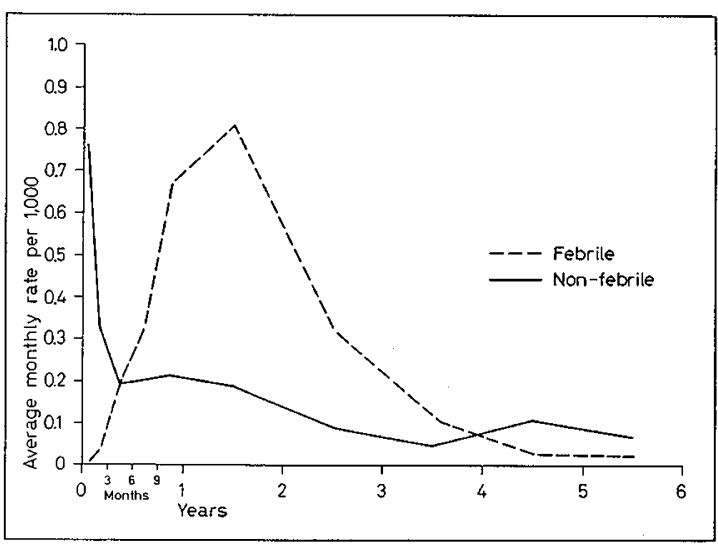

Fig. 1. Average monthly rates by age at first seizure of febrile and nonfebrile convulsions.

Child health and development studies, 1960-1967.

Table II. Incidence of febrile and nonfebrile convulsive disorders by age of first seizure. Child health and development studies 1960-1967

\begin{tabular}{|c|c|c|c|c|c|c|c|c|c|}
\hline \multirow[t]{2}{*}{ Age } & \multicolumn{3}{|c|}{$\begin{array}{l}\text { Children with } \\
\text { convulsions }\end{array}$} & \multicolumn{3}{|c|}{$\begin{array}{c}\text { Average monthly } \\
\text { incidence rates per } 1000\end{array}$} & \multicolumn{3}{|c|}{$\begin{array}{c}\text { Cumulative Incidence } \\
\text { rates per } 1000\end{array}$} \\
\hline & $F^{1}$ & $\mathrm{NF}^{2}$ & Total & $\mathrm{F}$ & NF & Total & F & $\mathrm{NF}$ & Total \\
\hline $0-<1$ month & - & 14 & 14 & - & 0.76 & 0.76 & - & 0.8 & 0.8 \\
\hline $1-<3$ months & 1 & 12 & 13 & 0.03 & 0.33 & 0.36 & 0.1 & 1.4 & 1.5 \\
\hline $3-<6$ months & 11 & 10 & 21 & 0.21 & 0.19 & 0.41 & 0.7 & 2.0 & 2.7 \\
\hline $6-<9$ months & 16 & 10 & 26 & 0.33 & 0.20 & 0.53 & 1.7 & 2.6 & 4.3 \\
\hline $9-<12$ months & 31 & 10 & 41 & 0.67 & 0.21 & 0.88 & 3.7 & 3.3 & 6.9 \\
\hline $1-<2$ years & 132 & 30 & 162 & 0.81 & 0.19 & 1.00 & 13.4 & 5.5 & 18.8 \\
\hline $2-<3$ years & 42 & 11 & 53 & 0.33 & 0.11 & 0.44 & 17.3 & 6.5 & 23.7 \\
\hline $3-<4$ years & 10 & 5 & 15 & 0.10 & 0.05 & 0.16 & 18.5 & 7.1 & 25.6 \\
\hline $4-<5$ years & 2 & 8 & 10 & 0.03 & 0.09 & 0.12 & 18.7 & 8.4 & 27.2 \\
\hline $5-<6$ years & 1 & 3 & 4 & 0.02 & 0.07 & 0.09 & 19.0 & 9.3 & 28.3 \\
\hline Total & 246 & 113 & 359 & & & & & & \\
\hline
\end{tabular}

${ }^{\mathrm{F}}=$ febrile convulsion

$2 \mathrm{NF}=$ nonfebrile convulsion 
experienced one or more febrile convulsions and one percent had a nonfebrile convulsion.

Since infections of the respiratory tract and otitis media, accompanied by high fever, are common in young children, it was not surprising that in the present study about $63 \%$ of the febrile convulsions were associated with these conditions. Thirteen percent were associated with roseola, and the remainder were associated with a variety of infectious diseases. Ten children or $4.0 \%$ of those with febrile convulsions had intracranial infections.

\section{Sex}

In the febrile group 131 (53.3\%) were boys and 115 (46.7\%) were girls. These percentages were not significantly different from those of the total cohort $(51.1 \%$ boys and $48.9 \%$ girls). Among the nonfebrile group, however, there was a statistically significant excess of boys $(61.9 \%$ boys; $\mathrm{p}<0.05)$.

\section{Birth Weight and Gestational Age}

Table III compares the distribution of the febrile and nonfebrile groups by birth weight and gestational age with that of the entire cohort of liveborn infants surviving the first month of life. The distribution of the febrile group by birth weight and gestational age was similar to that of the entire cohort. The nonfebrile group, however, had an excess of children with birth weights between 1500 and $2500 \mathrm{~g}$ (Groups II and III) compared with the entire cohort $(10.0 \%$ compared with $4.6 \% ; \mathrm{p}<0.05)$.

The excess was most pronounced in Group IIIcomposed of children with birth weights of 1500-2500 $\mathrm{g}$ and gestational ages of 37 or more weeks. Of the non- febrile children, $6.4 \%$ were in this birth weight-gestation group, while only $2.5 \%$ of the febrile children and $2.3 \%$ of the total cohort were in Group III. The children of birth weight-gestation Group III have been reported to have also a larger number of severe congenital anomalies [14]; this fact, however, does not explain entirely their high proportion of nonfebrile convulsions, because the excess is noted also when the children with severe anomalies were excluded.

\section{Multiple Episodes}

There was a wide range in the degree of severity of convulsive disorders, varying from a single almost accidental episode to very severe daily attacks. It was de-

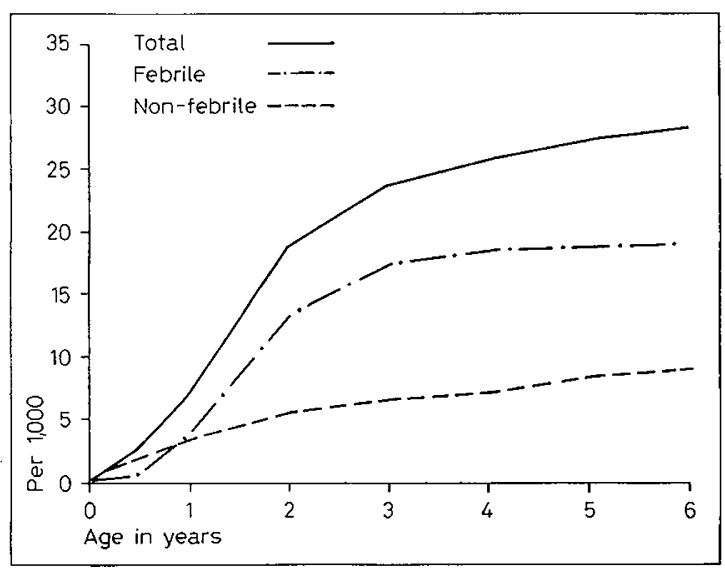

Fig. 2. Cumulative rates by age at first seizure of febrile and nonfebrile convulsions.

Child health and development studies, 1960-1967.

Table III. Percent distribution in 5 birth weight-gestation groups of children having febrile and nonfebrile convulsions. Child health and development studies, 1960-1967

\begin{tabular}{cccccc}
\hline Group & $\begin{array}{c}\text { Birth weight } \\
(\mathrm{g})\end{array}$ & $\begin{array}{c}\text { Gestational age } \\
\text { (weeks) }\end{array}$ & Febrile & Nonfebrile & $\begin{array}{c}\text { Cohort }^{1} \\
1960-1967\end{array}$ \\
\hline I & $\leq 1500$ & all gestations & 0.4 & - & 0.25 \\
II & $1501-2500$ & $<37$ & 1.6 & 3.6 & 2.25 \\
III & $1501-2500$ & $37+$ & 2.5 & 6.4 & 2.3 \\
IV & $>2500$ & $<37$ & 4.5 & 5.5 & 5.1 \\
V & $>2500$ & $37+$ & 91.0 & 84.5 & 90.1 \\
\hline & Total percent & & 100.0 & 100.0 & 100.0 \\
& I-V number & & 244 & 110 & 18,029 \\
\hline
\end{tabular}

\footnotetext{
1 Surviving the first month of life.

2 Excludes children for whom the birth weight-gestation group was not known.
} 
sirable, therefore, to differentiate between infants having multiple seizures and those with only a single attack. Multiple episodes occurred in one-third of the children in the febrile group and in three-quarters of those in the nonfebrile group. In a large proportion of the children who experienced multiple seizures, the second attack followed within less than a year of the first. The chance of the child having a second attack was independent of the child's age at which the first episode occurred. Of the 254 children who had an initial febrile seizure, eight or $3.1 \%$ later developed nonfebrile convulsions. It can also be stated that eight or $7.1 \%$ of the 113 children with nonfebrile convulsions had an initial febrile seizure.

The frequency of special diagnostic procedures increased greatly after the occurrence of a second convulsion; ninety-five percent of the children who experienced multiple episodes of convulsions were given an EEG examination.

\section{Severe Congenital Anomalies}

The procedure adopted by the Ghild Health and Development Studies in regard to congenital anomalies was first to ascertain the specific diagnostic entity in the necessary detail. For many of the analyses, however, the specific diagnostic entities were combined and investigated in three categories according to the severity of the anomaly: severe, moderate, and trivial. Some examples may clarify the categories. Severe anomalies included conditions that, if not corrected, would interfere seriously with the child's well being and/or development or were potentially hazardous. Examples are hypothyroidism, microcephaly, cleft lip, and congenital heart disease. Moderate anomalies represented the intermediate category and included conditions such as esotropia and exotropia, ptosis palpebrae, first degree hypospadias, polydactyly, and congenital dislocation of the hip. Trivial anomalies interfered only slightly, temporarily, or not at all with the child's health, and included metatarsus varus, skin tags, and blocked tear duct.

In the present paper, only the category of severe anomalies is reported.

A large proportion of the children in the nonfebrile group had severe congenital anomalies, especially anomalies of the central nervous system. Thirty-one percent of the children in the nonfebrile group and $7 \%$ of those in the febrile group had severe congenital anomalies (table IV). In comparison, only $3 \%$ of the children of the entire cohort who survived the first month of life suffered a severe congenital anomaly by age five. Thus, even among the febrile group, the frequency of severe congenital anomalies was significantly higher than in the entire cohort $(p<0.05)$.

In the febrile group, the frequency of severe congenital anomalies of the central nervous system was $1.2 \%$, a rate coming very close to that of the entire cohort. In the nonfebrile group, however, 30 children or $26.5 \%$ had such anomalies; seven of these children also had severe anomalies of other systems.

The frequency of severe congenital anomalies was much higher in children who experienced multiple episodes of seizures than in those who experienced only a single episode; however, even the latter had higher frequencies than is true for the entire cohort.

In the nonfebrile group, the frequency of cerebral palsy and of mental retardation was extremely high (table IV). Eleven or $9.7 \%$ of the children in the non-

Table IV. Number and percent of severe congenital anomalies in children having single or multiple episodes of febrile and nonfebrile convulsions

\begin{tabular}{|c|c|c|c|c|c|c|c|c|c|}
\hline \multirow[t]{4}{*}{ Convulsive disorder } & \multirow{4}{*}{$\begin{array}{l}\text { Number of } \\
\text { children }\end{array}$} & \multicolumn{8}{|c|}{ Severe congenital anomalies } \\
\hline & & \multicolumn{2}{|c|}{ Total } & \multicolumn{6}{|c|}{ of central nervous system } \\
\hline & & \multirow[b]{2}{*}{ No. } & \multirow[b]{2}{*}{$\%$} & \multicolumn{2}{|c|}{ Total } & \multicolumn{2}{|c|}{ Cerebral palsy } & \multicolumn{2}{|c|}{$\begin{array}{c}\text { Mental } \\
\text { retardation }\end{array}$} \\
\hline & & & & No. & $\%$ & No. & $\%$ & No. & $\%$ \\
\hline Febrile total & 246 & 18 & 7.3 & 3 & 1.2 & 2 & 0.8 & - & - \\
\hline 1 episode & 172 & 11 & 6.4 & 3 & 1.7 & 2 & 1.2 & - & - \\
\hline 2 or more episodes & 74 & 7 & 9.5 & - & - & - & - & - & - \\
\hline Nonfebrile total & 113 & 35 & 31.0 & 30 & 26.5 & $11^{1}$ & 9.7 & $18^{1}$ & 15.9 \\
\hline 1 episode & 40 & 7 & 17.5 & 5 & 12.5 & 1 & 2.5 & 3 & 7.5 \\
\hline 2 or more episodes & 73 & 28 & 38.4 & 25 & 34.2 & 10 & 13.7 & 15 & 20.5 \\
\hline
\end{tabular}

${ }^{1}$ Include 6 who had both cerebral palsy and mental retardation. 
febrile group had cerebral palsy and 18 or $15.9 \%$ were mentally retarded. These figures include 6 children who had cerebral palsy as well as mental retardation. Thus, 23, or one-fifth of the 113 children in the nonfebrile group, had cerebral palsy or mental retardation or both. This figure may be compared with $1.2 \%$ in the febrile group and $0.5 \%$ in the cohort. The number of mentally retarded children is understated since many of the children were still too young for a reliable estimate of their mental development.

The relation of anomalies of the central nervous system to febrile and nonfebrile convulsions may be considered in another way. In the cohort there were a total of 165 children with severe anomalies of the central nervous system. In these children, the incidence of febrile convulsions was the same as that of the total cohort $(1.8 \%)$, while the incidence of nonfebrile convulsions was ten times as high $(18.2 \%)$.

Among the 165 children there were 34 with cerebral palsy and 62 with mental retardation, including 12 with both conditions. Thus, the incidence of a nonfebrile convulsion was $32.3 \%$ for children with cerebral palsy, $29.0 \%$ for mentally retarded children, and $50.0 \%$ for children with both conditions.

\section{Mortality}

There was a relatively high mortality of children with convulsive disorders, especially of those in the nonfebrile group. There were four deaths among the 246 children with febrile disorders and eight deaths among the 113 children with nonfebrile disorders. Since all children had passed the neonatal period, this number of deaths exceeded the expected number ( 4 versus $1 \frac{1}{2}$ after the first month of life in the febrile group and 8 versus less than 1 in the nonfebrile group). This excess does not necessarily reflect an extra risk of death associated with convulsions per se, but may reflect the high level of mortality of the associated conditions. For example, in the nonfebrile group, the eight children who died had severe congenital anomalies.

In the febrile group, two children died from the infection that triggered the initial seizure, one from a general infection of unknown origin and one from viral encephalitis. The third child died of complications of a congenital anomaly (biliary atresia) and the fourth child had a fatal accident.

\section{Discussion}

This study provided the opportunity to observe and study children with convulsive disorders in the setting of an entire cohort of which they were a part. It was possible to obtain quantitative expressions for certain phenomena, some of which confirmed previous estimates derived clinically, and others of which have not been reported. The derived incidence rate of $3 \%$ of convulsive disorders by five years of age confirmed estimates obtained clinically by other investigators [4, $7,11]$. More refined estimates of the incidence rate by age at the time of the first seizure of febrile and nonfebrile disorders are provided.

The peak risk of a first convulsion in the nonfebrile group occurred during the first month of life, while that for the febrile group occurred during the second year of life. Since the incidence of acute infections has been found to be as high during the first as it is during the second year of life [13], it is suggested that the risk of febrile convulsions is not entirely a function of the frequency of acute infections.

The method used in the present study provides quantitative expressions for the risk of repeated attacks. The percentages of recurrence of seizures reported by other investigators $[2,6,9,12]$ vary widely, and are, in general, higher than those of this study. Quantitative expressions were derived also for the relation of convulsive disorders to congenital anomalies, anomalies of the central nervous system, cerebral palsy and mental retardation. While this relation has been commented on before, no specific figures have been reported $[3,10]$.

In the present study, $3 \%$ of the children who had an initial febrile seizure had later nonfebrile convulsions. This percentage is of the same order of magnitude as that reported as 'simple febrile disorders' by Livingston [9] and those reported by Friderichsen and Melchror [5] and by Mrlzichap [12].

The role of prematurity in convulsive disorders can be adequately studied when the distribution by birth weight and gestational age is compared with that of the entire cohort. Febrile convulsions appeared as frequently among full term infants of 'normal' birth weight as among children who were born preterm or with low birth weights. Nonfebrile convulsions, however, appeared twice as frequently among infants of low birth weight; a similar excess in the incidence of low birth weight has been reported for children with epilepsy [8]. Our data indicate that the risk of nonfebrile seizures is especially high for infants of low birth weight and gestational age of 37 or more weeks. It is important to observe, however, that more than $85 \%$ of the children with nonfebrile convulsions were 'mature' by both birth weight and gestational age criteria.

\section{Summary}

This study is based on all children ascertained as having had convulsive disorders in a cohort of some 18,500 
children; 246 children had febrile convulsions and 113 had nonfebrile convulsions. The risk of a convulsive disorder by five years of age was found to be $2 \%$ and $1 \%$ for febrile and nonfebrile seizures respectively. The highest incidence of the initial seizure for children of the febrile group occurred during the second year of life, and for children of the nonfebrile group during the first month of life. Nearly one-third of the children in the febrile group and two-thirds of the children in the nonfebrile group had multiple episodes of seizures. About $3 \%$ of the children having initial febrile seizures later had nonfebrile convulsions.

The risk of febrile seizures was about equal for boys and girls, but relatively more boys than girls suffered nonfebrile seizures. In the febrile group, the relative frequency of low birth weight children was similar to that of the total cohort, but the nonfebrile group had an excess of low birth weight children, especially those whose gestational age was 37 or more weeks. The frequency of severe congenital anomalies was $7 \%$ in the febrile group and $31 \%$ in the nonfebrile group, compared with $3 \%$ for the entire cohort. The majority of severe anomalies in the nonfebrile group were those of the central nervous system, especially manifested as mental retardation and cerebral palsy.

\section{References and Notes}

1. BREG, W.R. and YANNET, H.: The child in a convulsion. Pediat. Clin. N. Amer. 9: 101-112 (1962).

2. CArter, S.: Diagnosis and treatment: Management of the child who has had one convulsion. Pediatrics 33: 431-434 (1964).

3. Dennhoff, E. and Robinault, I.P.: Cerebral palsy and related disorders, a developmental approach to dysfunction (McGraw-Hill, New York/ Toronto/London 1960).

4. FARMER, T.W.: In Pediatric Neurology (ed. FARMER, TH.W.), chapter 2, pp.65-108 (Harper and Row, New York 1964).
5. Friderichsen, C. and Melchior, J.: Febrile convulsions in children, their frequency and prognosis. Acta pediat. Scand. 43: (Suppl. 100) 307-317 (1954).

6. Lennox, W. G.: Significance of febrile convulsions. Pediatrics 11: 341-357 (1953).

7. Lennox, W.G.: Epilepsy and related disorders, vol. 1 (Little, Brown, Boston/Toronto 1960).

8. Lilienfeld, A.M. and Pasamanick, B.: Association of maternal and fetal factors with the development of epilepsy. 1. Abnormalities in the prenatal and paranatal periods. J.amer.med.Ass. 155: 719-724 (1954).

9. Livingston, S.: Convulsive disorders in infants and children. Advanc. Pediat., vol. 10, pp. 113-195 (Year Book Publishers, Chicago, Ill. 1958).

10. Mason, T. and Niedermeyer, E.: Convulsive disorders during the first three months of life. Epilepsia 9: 1-9 (1968).

11. Millichap, J.G.: Diagnosis and management of convulsive disorders with special emphasis on seizures amenable to specific therapies. Pediat. Clin. N. Amer. 7: 583-608 (1960).

12. Millichap, J. G.: Febrile convulsions (Macmillan, New York 1968).

13. VAN DEN BERG, B.J.: Morbidity of low birth weight and/or preterm children compared to that of the 'Mature'. I. Methodological considerations, and findings for the first two years of life. Pediatrics 42: 590-597 (1968).

14. Yerushalmy, J.; van den Berg, B.J.; Erhardt, C. L. and JACOBZINER, H. : Birth weight and gestation as indices of 'Immaturity' neonatal mortality and congenital anomalies of the 'immature'. Amer. J. Dis. Child. 109: 43-57 (1965).

15. Supported by PHS Grant No. HD-00718 of the National Institutes of Health, U.S. Public Health Service.

16. Requests for reprints should be addressed to: Dr. Bea J. van den Berg, Child Health and Development Studies, University of California, Berkeley 3867 Howe St., Oakland, Cal. 94611, USA. 